\title{
The Ultrasound Appearance of Early Periventricular Leukomalacia with Neuropathologic Correlates
}

\author{
Asma Q. Fischer M.D., \\ Joseph C. Anderson M.D., \\ Robert M. Shuman, M.D.
}

DOI: http://dx.doi.org/10.5915/17-1-12734

\begin{abstract}
Periventricular leukomalacia is a necrotic lesion of the cerebral white matter. It is seen in premature infants weighing $1000-1900$ grams at birth, who have cardiorespiratory disturbances early in life and survive more than a week. It is characterized by necrosis of the periventricular axons adjacent to the lateral margins of the lateral ventricles. Premorbid recognition of this entity has been facilituted by the use of cranial ultrasound. Two types of lesions have been described sonographically: porencephalic and hemorrhagic. The former is anechoic and the latter hyperechoic; both are located periventricularly.

We present a third type of sonographic pattern associated with early, non-hemorrhagic periventricular leukomalacia. This sonographic pattern differs from those previously described. The cranial ultrasound and neuropathologic findings will be presented in two premature infants.
\end{abstract}

Periventricular leukomalacia is a necrotic lesion of the cerebral white matter. It is seen in premature infants weighing 1000-1900 grams at birth, who have cardiorespiratory disturbances early in life and survive more than a week ${ }^{1,2}$. It is characterized by necrosis of the periventricular axons adjacent to the lateral margins of the lateral ventricles.

Premorbid recognition of this entity has been facilitated by the use of cranial ultrasound. Two types of lesions have been described sonographically: porencephalic and hemorrhagic. The former is anechoic and the latter hyperechoic; both are located periventricularly $y^{3,4}$. We present a third type of sonographic pattern associated with early, nonhemorrhagic periventricular leukomalacia. This sonographic pattern differs from those previously described. The cranial ultrasound and neuropathologic findings will be presented in two premature infants. Hemorrhagic periventricular leukomalacia, subependymal hemorrhage with intraparenchymal extension, and early periventricular leukomalacia may all appear hyperechoic on sonography. Points of differentiation between these entities will be discussed.

\section{Case 1: S.M.}

The patient was a 29 week gestation male infant with a birth weight of 940 grams born of a 22 year old, primigravida following premature rupture of membranes with placenta praevia. Apgar scores were 3 and 5 at 1 and 5 minutes. He responded well to resuscitation but developed severe respiratory distress syndrome and needed continuous ventilatory support throughout his life. He had an initial leukocytosis for which a definite cause could not be ascertained despite a complete workup for sepsis. His patent ductus arteriosus closed

From the Departments of Neurology and Radiology.

The Medical College of Georgia, Augusta, Georgia

The University of Nebraska Medical Center, Omaha,

Nebraska and the University of Kentucky, Louisville, Ky.

Address all correspondence to Dr. Asma Q. Fischer, Section

of Pediatric Neurology, CK 290, Medical College of Georgia.

Augusta, Georgia, 30912. spontaneously at 9 days of age. At 15 days of age he had a severe episode of respiratory distress and bradycardia. He developed atelectasis of his right upper lobe, and had recurrent episodes of respiratory distress for the remainder of his life. At 20 days of age he developed necrotizing enterocolitis and underwent bowel resection. He continued to deteriorate clinically and a second bowel resection including the transverse colon up to the mid-jejunum was performed. Extensive necrosis of the bowel was present. At 23 days of age he died of cardiorespiratory arrest.

\section{Cranial ultrasound findings:}

The initial ultrasound scan done on the first day of life showed a small left subependymal hemorrhage and normal sized ventricles. The second ultrasound scan done at 4 days of age showed subependymal hemorrhage with intraventricular hemorrhage in the left lateral ventricle and a hyperechoic region periventricularly (Fig. 1 and 2 ). There was no evidence of ventricular enlargement. The right side was normal. Ultrasound scans at 15 and 17 days of age were unchanged.

\section{Neuropathologic Findings:}

The brain weighed 150 grams. The-substance of the occipital radiations was rarefied and shrunken, most marked at the dorsolateral angle of the left lateral ventricle at the level of the trigone (Fig. 3 and 4). Light microscopy showed tissue rarefaction in the periventricular zone of the left occipital radiation. Glial and vascular proliferation, axonal spheroids, and macrophages representative of an extensive zone of periventricular leukomalacia were also observed. There was no hemorrhage of the left germinal matrix, with rupture of the rostral margin of the foramen of Monro filling the ventricles with blood.

\section{Case 2: F.H.}

F.H. was the product of a 26 weeks pregnancy complicated by premature labor at 25 weeks, interrupted by placement of cerclage and administration of Ritodrine. The Ritodrine was stopped when the mother developed congestive heart failure. Labor progressed, and the pa- 


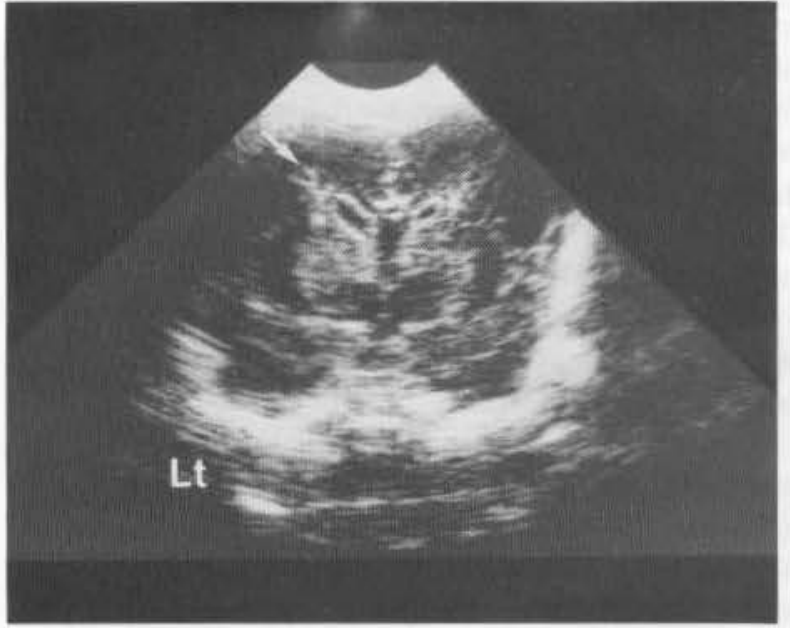

Fig. 1: Case 1. Cranial ultrasound in the mid-coronal plane. Arrow shows the streaky. irregular lesion in the periventricular region.

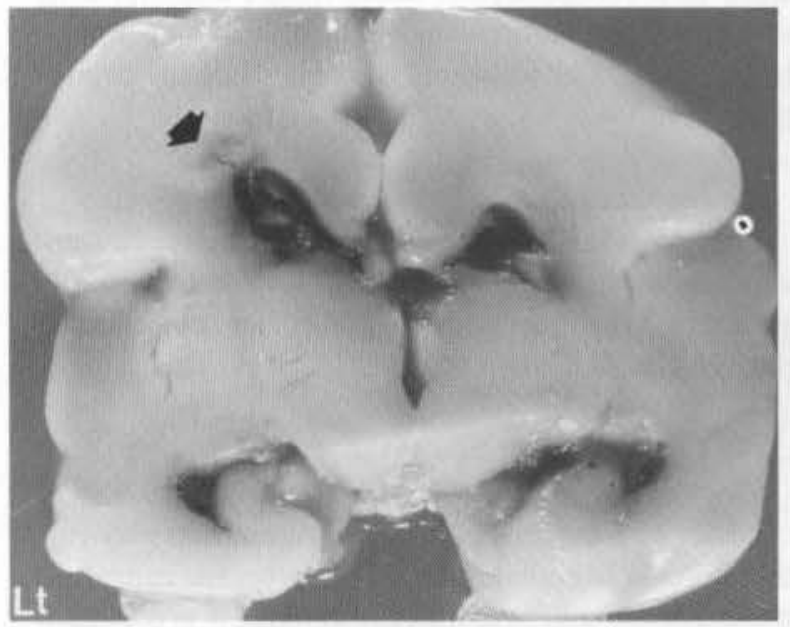

Fig. 3: Case 1. Mid-coronal section in the sonographic plane. Arrow shows periventricular leukomalacia.

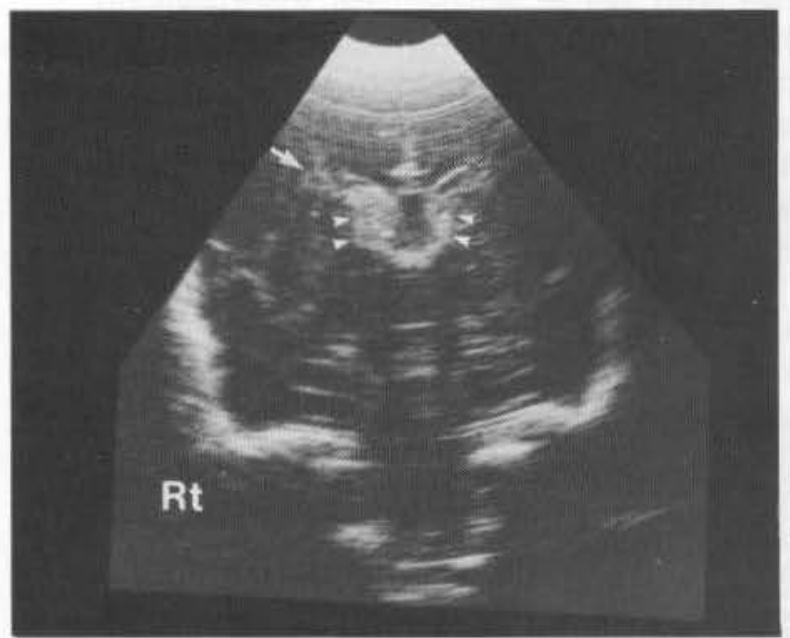

Fig. 5: Case 2. Cranial ultrasound in the coronal plane. Arrow shows an irregular periventricular hyperechoic lesion. Arrowheads show the the subependymal hemorrhage.

tient, weighing 1020 grams, was delivered by cesarean section. Apgar scores were 4 at 1 minute and 2 at 5 minutes. The patient was resuscitated and transferred to the University of Nebraska Medical Center. From the time of transfer the patient required continuous

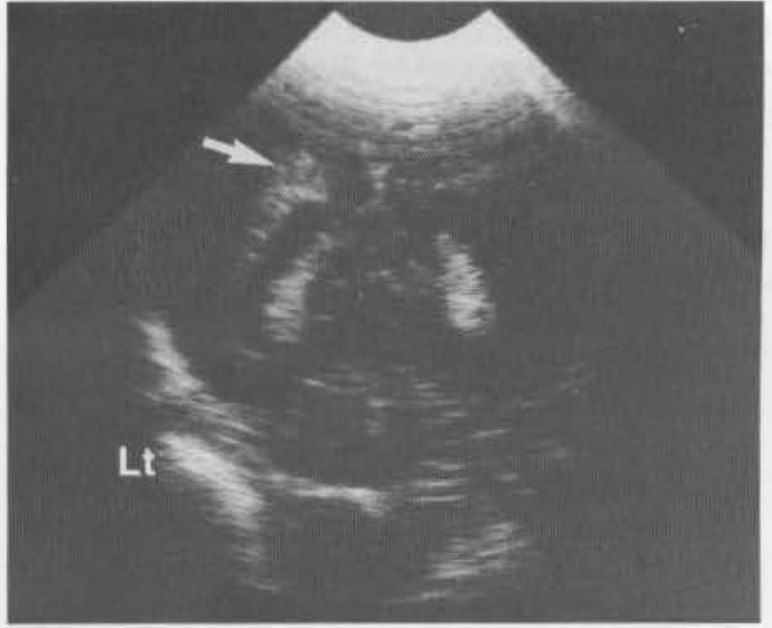

Fig. 2: Case 1. Cranial ultrasound in the posterior coronal plane. Arrow shos the irregular hyperechoic lesion in periventricular region of the trigone.

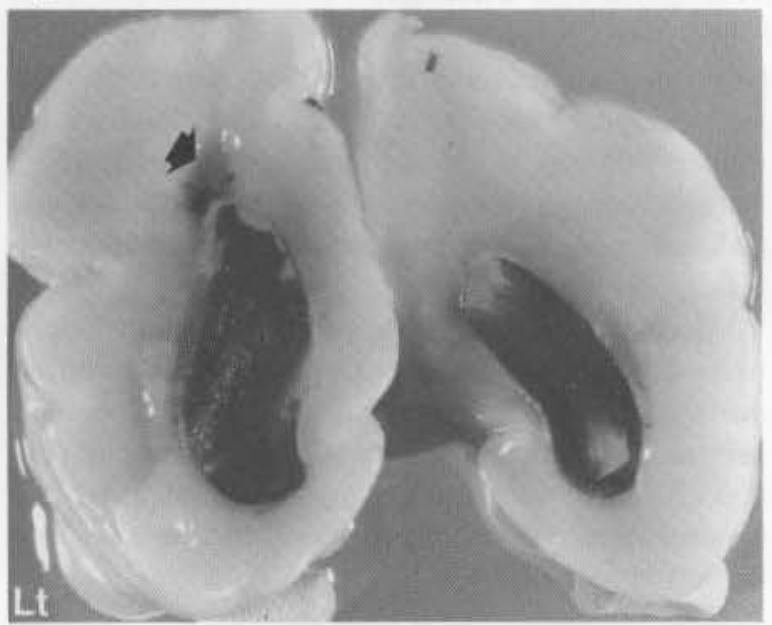

Fig. 4: Case 1. Posterior coronal section in the sonographic plane. Arrow shows periventricular leukomalacia.

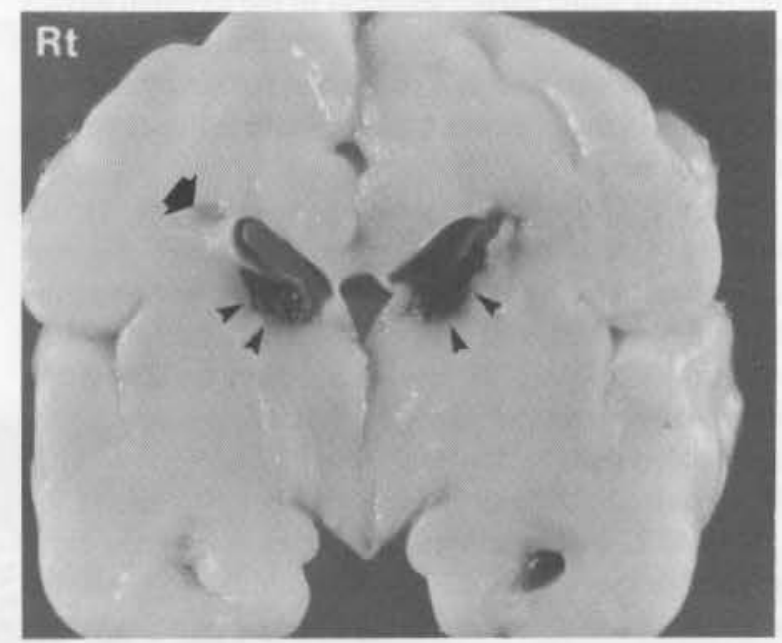

Fig. 6: Case 2. Coronal section in the sonographic plane. Arrow shows periventricular leukomalacia. Arrowheads show organizing subependymal hemorrhages.

mechanical ventilatory support. At 2 days of age she developed severe interstitial emphysema, which was treated with decompression. She had a patent ductus arteriosus which was treated with Indomethacin until 4 days of age. At 7 days of age she had an acute episode 
of hypoxia and acidosis and developed oliguria and azotemia which persisted for the remainder of her life. There was progressive deterioration in her pulmonary and renal function and her cardiac output decreased. At 16 days of age she finally succumbed to a bradycardic episode which progressed to a cardiopulmonary arrest.

\section{Cranial Ultrasound Findings:}

A cranial ultrasound scan was done at 2 days of age. It showed bilateral subependymal hemorrhage, (Grade I). A second ultrasound scan at 10 days of age showed mild ventricular dilatation with no change in the subependymal hemorrhages noted earlier. A hyperechoic region was noted in the right periventricular region but was not categorized as a definite pathologic entity (Fig. 5).

\section{Neuropathologic Findings:}

The fixed brain weighed 128 grams. There was periventricular leukomalacia of the lower half of the right occipital radiation, extending caudally from the trigone half way to the occipital pole (Fig. 6). There were similar but less severe lesions of periventricular leukomalacia affecting the occipital radiation of the left hemisphere.

Microscopically there was extensive zones of bland necrosis, macrophages, capillary proliferation, and axonal spheroids in the right occipital radiation. The whole periventricular region was notable for its vascular congestion. There were bilateral organizing subependymal hemorrhages, the left one of which had ruptured into the left lateral ventricle.

\section{Discussion:}

Periventricular leukomalacia is characterized by necrosis of white matter adjacent to the external angles of the lateral ventricles. The essential feature of periventricular leukomalacia is a presumed failure of cerebral perfusion in the presence of demonstrable impaired cardiac function ${ }^{2}$.

In Case 1, the patient developed severe respiratory distress syndrome and needed ventilatory support throughout life. A periventricular hyperechoic area was identified at 4 days of age, and became clearly evident sonographically by 15 days of age. On reviewing the neuropathic material the periventricular hyperechoic area correlated with the zone of periventricular leukomalacia, without evidence of hemorrhage.

In case 2 , the asymmetric periventricular hyperechoic region was seen at 10 days of age after the patient had experienced marked cardiorespiratory dysfunction. As in the preceding case, the asymmetric hyperechoic region correlated with the asymmetric periventricular leukomalacia.

Extravasated whole blood and blood in the stage of clot formation and retraction appears hyperechoic on sonography ${ }^{5,6}$. The periventricular hyperechoic regions in periventricular leukomalacia may reflect the neuropathologic state. Capillary proliferation, periventricular hemostasis and congestion, axonal spheroids and mineralization of the necrotic neurites may all provide multiple interfaces, for the echoes causing the lesion to be hyperechoic. Early periventricular leukomalacia is sonographically irregular, asymmetric and markedly hyperechoic in the trigonal periventricular regions. These characteristics differentiate it from the minimal asymmetric echoes seen in similar regions in presumably normal premature brains, and from the generalized hyperechogenecity seen in term infants with post asphyxial encephalopathy?

Extension of a subependymal hemorrhage into the region of the trigone starting at the level of the foramen of Monro mimics the hyperechoic lesion of periventricular leukomalacia. The outcomes of the two entities are different. Thus it is important to be aware that all hyperechoic lesions in the periventricular parenchyma of a premature infant are not intraparenchymal extensions of a subependymal hemorrhage, and that early stages of periventricular leukomalacia may appear hyperechoic. The following facts may help in differentiating the two:

\section{(a) Gestational age:}

Subependymal hemorrhages originate or extend caudally at the trigone in infants less than 28 weeks gestation. However, such infants do not develop periventricular leukomalacia ${ }^{2}$.

\section{(b) Locus and contiguity:}

Extensive subependymal hemorrhage may extend caudally into the cerebrum at the trigone and may rupture into the lateral ventricle. However, the caudal extension of this subependymal hemorrhage is in continuity with the subependymal hemorrhage at the level of the foramen of Monro. When a discontinuity between these two areas can be demonstrated sonographically, illustrated by alternating hyperechoic and isoechoic areas between the foramen of Monro and trigone in infants of 28 weeks gestation or more, the statistical probabilities are in favour of a subependymal hemorrhage at the foramen of Monro with a coexistent periventricular leukomalacia at the trigone. (See table 1).

Computerized cranial tomography may help in differentiating a hemorrhage from periventricular leukomalacia as the hemorrhage would appear dense and the periventricular leukomalacia lucent.

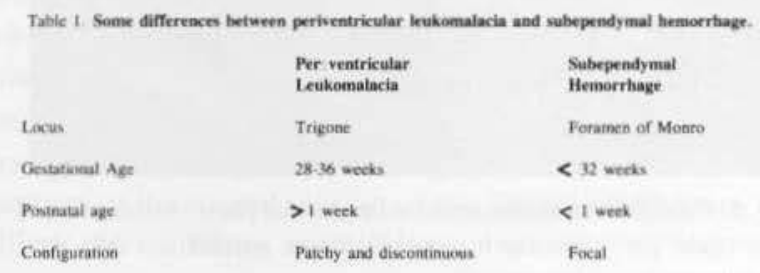

\section{REFERENCES}

1. Larroche JCL: Developmental Pathology of the Neonate. Amsterdam, Elsevier, Norten Holland, 1977

2. Shụman RM and Selednik LJ: Periventricular leukomalacia. A one year autopsy study, Arch 
Neurol, 37:321, 1980

3. Hill A, Tzipora D, et al: Diagnosis and evolution of periventricular leukomalacia. A study with realtime ultrasound. Abstracts of the Eleventh Child Neurology Society Meeting, October 1982.

4. Hill A, Melson GL, et al: Hemorrhagic periventricular leukomalacia: Diagnosis by realtime ultrasound and correlation with autopsy findings.

Pediatrics, 69:282, 1982
5. Grant EJ, Schellweger D, Boots FT, et al: Realtime sonography of the neonatal and infant head. Am J Rad 136:265, 1981

6. Anderson JC, Baltaxe HA, et al: Inability to show clot: One limitation of ultrasonography of the abdominal aorta. Radiology, 132:693, 1979

7. Babcock DS, Ball W: Postasphyxial encephalopathy in full term infants: Ultrasound diagnosis. Radiology, 148:423, 1983 